\title{
Spatiotemporal properties of nanoshell plasmonic response for strong-field experiments
}

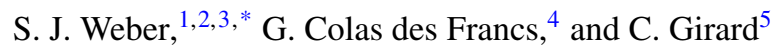 \\ ${ }^{1}$ CEA-Saclay, IRAMIS, Laboratoire interactions dynamique et lasers, 91191 Gif-sur-Yvette, France \\ ${ }^{2}$ Université de Toulouse; UPS; Laboratoire Collisions Agrégats Réactivité, IRSAMC; F-31062 Toulouse, France \\ ${ }^{3}$ CNRS; UMR 5589; F-31062 Toulouse, France \\ ${ }^{4}$ Laboratoire Interdisciplinaire Carnot de Bourgogne (ICB), UMR 6303 CNRS-Université de Bourgogne, 9 Avenue A. Savary, \\ BP 47 870, F-21078 Dijon, France \\ ${ }^{5}$ Centre d'Elaboration de materiaux et d'Etudes structurales (CEMES), CNRS, 29 rue J. Marvig, BP 94347 , \\ F-31055 Toulouse Cedex 4, France
}

(Received 7 February 2015; revised manuscript received 26 April 2015; published 14 May 2015)

\begin{abstract}
Field enhancement behavior of a $\mathrm{SiO}_{2} / \mathrm{Au}$ nanoshell is studied in the framework of strong-field physics. Localized plasmonic fields induce local electric field enhancement with the potential to lead to the strong-field regime without the use of costly amplified lasers. In this framework, electrons are tunnel ionized from the nanoshell and accelerated by the local field being spatially inhomogeneous in terms of spectral and polarization properties. These processes are happening within a single laser shot, and thermal effects are therefore neglected. We show that the localized response to ultrashort femtosecond pulses can be investigated by extending Mie theory to multilayer spherical particles. Nanoshell plasmonic resonances can be easily tuned depending on the volume ratio between core and whole particle. Optimum geometric parameters of the nanoshell are selected for use with ultrashort pulses centered at $800 \mathrm{~nm}$ where most femtosecond oscillators operate. It is shown that phase and amplitude reshaping of the incident pulse by the plasmonic resonance can be partially corrected using active shaping leading to ultrashort response of the medium. Finally, free electron classical trajectories are calculated to highlight the inhomogeneous nature of the local enhancement in a strong-field picture.
\end{abstract}

DOI: 10.1103/PhysRevB.91.205419

PACS number(s): 78.67.Bf, 78.67.Pt, 42.25.Ja, 79.60.Jv

\section{INTRODUCTION}

The investigation of the collective oscillations of conduction electrons in metallic nano-objects, the so-called plasmon modes [1], is at the convergence of many fields of research [2-5]. Plasmons propagation properties open up possibilities to design subwavelength optical circuitry [6] while increased plasmonic control applies to quantum optics [7], energy transfer [8], and many other possibilities. Another widely used property is the huge local field enhancement occurring when plasmons are excited near the resonance. Factors up to a few orders of magnitude on the field can be achieved using plasmonic antennas [9]. Surface-enhanced Raman spectroscopy (SERS) took advantage of this for molecular sensing now reaching the level of single molecule detection $[10,11]$.

Plasmonic field enhancement met strong-field physics in 2006 with either direct photo-emission from the nano-object or high harmonic generation (HHG) [12] from gas phase atoms surrounding the nano-object. The interest in this physics is related in both cases to the complex electron trajectories in a highly inhomogeneous electric field, contrary to standard gas phase strong-field physics. The latter is characterized by a valence shell electron dynamic driven by a homogeneous intense laser field and may be decomposed in three phases. This so-called three step model [13-15] consists first of a tunnel ionization within a laser subcycle followed by a quiver motion of the electron in the homogeneous electric field. The recollision of the electron on its parent ion leads to

\footnotetext{
*sebastien.weber@irsamc.ups-tlse.fr
}

either electron scattering or recombination with emission of an energetic photon in the XUV range in the form of an extended harmonic spectrum [16]. In the presence of spatially confined plasmonic fields, this picture is modified, and the resulting inhomogeneous electric field generates more complex electron trajectories. Direct photoemission from a nano-object has been demonstrated with the first laser induced photoemission from nanotips [17] on a subcycle timescale. It has been shown that the strong-field regime can be reached using "simple" femtosecond oscillators. This regime is characterized by the onset of field driven electron recollisions, leading to higher photoelectron energy emission in a femto- to subfemtosecond time range [18]. The influence of inhomogeneities has been thoroughly investigated on gold nanotips excited with short pulses ranging from the near IR to midIR [19]. Concerning the plasmonic assisted HHG, Kim et al. used bowtie nanoantennas [20] to trigger this highly nonlinear process on surrounding gas phase atoms. However, while field enhancement was present and sufficient, the chosen geometry could not produce phase matched harmonics except for atomic fluorescence [21,22]. Researchers are now looking for other schemes and geometries to trigger plasmonic assisted HHG [23]. Following these papers, many theoretical treatments have been devoted to the description of photoemission and high harmonic generation in spatially inhomogeneous fields [24,25] with the possibility of using the enhancement as a degree of freedom [26].

Despite this wealth of research, most of the studies highlight only the local field amplitude enhancement. We propose here a complete study of the local field response to ultrashort femtosecond pulses in terms of amplitude, phase, and polarization. The local amplitude set the required laser intensity to reach strong fields $\left(\approx 10^{14} \mathrm{Wcm}^{-2}\right)$. The amplitude 
and spectral phase of the resonance can induce field reshaping, and the amplitude and polarization properties are important in terms of electronic trajectories. In this context, hybrid nano-objects, so called nanoshells, consisting of a dielectric core and a metallic shell placed in vacuum are investigated. These objects have a tunable resonance particularly suited to the study of wavelength impact on strong-field experiments in inhomogeneous fields. Moreover, small spherical particles can be used in a jet of nanoparticles [27] under vacuum. Tuning the particle density could lead either to the isolated particle regime or to dense jet. Using isolated particle removes the requirement for spatially resolved experiments. On the contrary, dense jet may provide sufficient emitting surrounding atoms to induce measurable HHG. Moreover, particles used in a jet are replaced for each laser shot. Long term thermal stability is automatically satisfied, and damage threshold can be exceeded without target replacement issues. Such an experiment as been demonstrated recently on strong-field photoionization from silica spherical particles [27] delivered in vacuum. For these reasons, dielectric/metallic nanoshells are an ideal object for strong-field studies.

In this paper, by applying the Mie theory extended to multilayer systems [28], we investigate the behavior in space, time, and polarization of the electric local field induced by a short light pulse on a $\mathrm{SiO}_{2}$ /gold nanoshell placed in vacuum. This framework is particularly adapted for spherical multilayer particles [29] and has less computer requirement compared to other numerical techniques [30]. The nanoshell behavior is looked at as a function of wavelength and geometry in order to obtain ideal parameters for strong-field experiments such as: plasmonic resonance in the near-IR and spectrally broad enough to not induce too much field reshaping; higher decreasing field length compared to electron excursion. The impact of the resonance on the spatial and temporal profile is highlighted, and we show that active spectral field shaping can be used to correct the temporal effects. Finally, the impact of intensity and polarization inhomogeneities on classical trajectories of electrons born near the particle surface are looked at as a function of incident field wavelength. These conditions simulate the behavior of electrons produced from either photoemission from the shell or tunnel ionization from atoms lying close to the particle. Both are the first step toward high harmonic generation.

\section{METHODS}

We consider a $\mathrm{SiO}_{2} /$ gold nanoshell placed in vacuum as defined in Fig. 1 and of, respectively, radius $a_{2}$ and $a_{1}$. The geometrical parameter $\beta=\left(a_{2} / a_{1}\right)^{3}$ will be used in the following to quantify the ratio between the core and the whole particle volume. This particle is excited by an incident plane wave electric field polarized along $\mathbf{u}_{\mathbf{x}}$ and propagating along $\mathbf{u}_{\mathbf{z}}$. It is defined as

$$
\mathbf{E}=E_{0} \exp [-i(\omega t-k z)] \mathbf{u}_{\mathbf{x}},
$$

where $E_{0}$ is the incident laser electric field amplitude, $\omega$ the carrier frequency, and $k$ the amplitude of its wave vector oriented along $(\mathrm{Oz})$.

The local field response to a monochromatic excitation is calculated using extended Mie theory for multilayer spherical

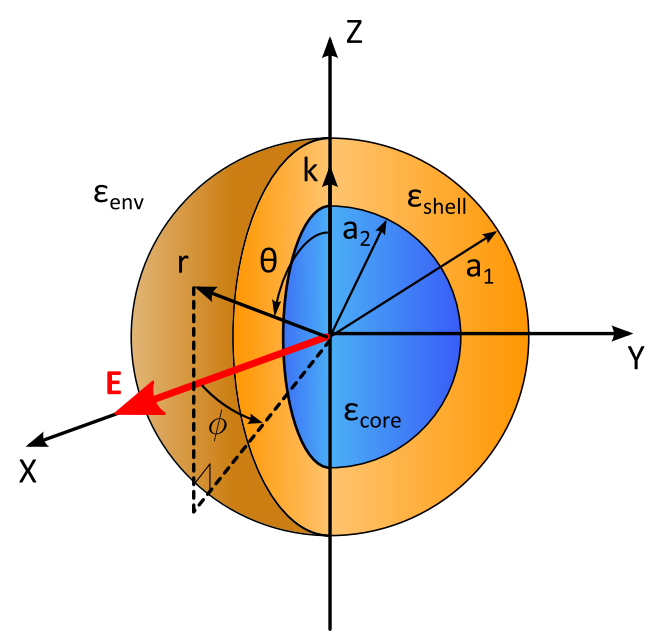

FIG. 1. (Color online) Layout of a nanoshell excited by a incident electric field polarized along $\mathbf{u}_{\mathbf{x}}$ and propagating along $\mathbf{u}_{\mathbf{z}}$. Shell and core radii are respectively $a_{1}$ and $a_{2}$ while their permittivities are $\varepsilon_{\text {shell }}$ and $\varepsilon_{\text {core }}$. A medium of permittivity $\varepsilon_{\text {env }}$ is surrounding the nanoshell.

particles [28,31]. The wave equation governing the interaction of the incident field and the nanoshell response is solved in spherical coordinates where the solutions can be expressed as an expansion of a linear combination of spherical vector harmonics. Numerical calculation includes a given number of modes making the solution accurate for particles of arbitrary size. The coefficients of the expansion are obtained by solving a set of linear equations in each material of the particle. They are obtained from the continuity of the fields at the core/shell/environment boundaries.

The environment permittivity is set to unity (nanoshell in vacuum). The $\mathrm{SiO}_{2}$ dielectric core permittivity has been calculated from its refractive index using a Sellmeier equation. This last is nearly constant, equal to $n_{\text {core }}=1.45$ in the wavelength range considered (400-1800 nm). Permittivities of metallic shells have been taken from experimental data [32] corrected to take into account electron scattering at the shellenvironment interface. When $\beta$ is increasing the metallic shell thickness becomes small compared to the electron mean free path (typically $42 \mathrm{~nm}$ in bulk gold). Damping of the dielectric response happens due to scattering of these electrons on the inner and outer metallic surfaces, limiting the enhancement and broadening the plasmonic resonance depending on the shell size and environment permittivity [33]. This effect can be phenomenologically taken into account by modifying the permittivity response of considered metals. The collisional frequency of the bulk $\gamma_{\text {bulk }}$ is modified using Mathiessen's rule and Billiard theory [34]:

$$
\Gamma(\bar{x})=\gamma_{\text {bulk }}+\frac{V_{F}}{\bar{x}},
$$

where $V_{F}$ is the Fermi velocity and $\bar{x}$ is the size dependent mean free path in the nanoshell given by

$$
\bar{x}=\frac{4}{3}\left(\frac{a_{1}^{3}-a_{2}^{3}}{a_{1}^{2}+a_{2}^{2}}\right)=\frac{4 a_{1}}{3}\left(\frac{1-\beta}{1+\beta^{2 / 3}}\right) .
$$

As expected, this equation shows that for a given volume ratio $\beta$, the effect will be less important when the shell radius $a_{1}$ 
increases, tending to the bulk expression. Using Drude theory, this damping is taken into account with [35]

$$
\varepsilon_{\text {shell }}(\bar{x}, \omega)=\varepsilon_{\text {shell }}^{\exp }(\omega)+\frac{\omega_{p}^{2}}{\omega^{2}+i \omega \gamma_{\text {bulk }}}-\frac{\omega_{p}^{2}}{\omega^{2}+i \omega \Gamma(\bar{x})},
$$

where $\omega_{p}$ is the plasma frequency.

The local response to an ultrashort femtosecond pulse is calculated by coherently adding monochromatic plane waves in the considered wavelength range. These spectral contributions are all calculated for a normalized incident electric field. It is therefore trivial to simulate a spectral profile weighting each calculated contribution by a spectral field:

$$
\widetilde{E}(\omega)=A(\omega) e^{i \phi(\omega)},
$$

where $A(\omega)$ is the spectral amplitude and $\phi(\omega)$ is the spectral phase, constant for a transform limited pulse [36]. The profile of the spectral amplitude depends mostly on the conditions under which the short pulse is produced. We choose here a Gaussian envelope for the amplitude,

$$
A(\omega)=\exp \left[-2 \ln 2\left(\frac{\omega-\omega_{0}}{\Delta \omega}\right)^{2}\right],
$$

defined by a central frequency $\omega_{0}$ and a spectral FWHM in intensity $\Delta \omega$. That profile is close to a femtosecond oscillator spectral shape. The possible amplitude and phase shapes are not limited. Using a pulse shaper [37], both the amplitude and phase of the incident pulse could be modified in order to control the local field response as will be shown in Sec. III B.

\section{NANOSHELL OPTICAL FIELD BEHAVIOR STUDIED FROM THE EXTENDED MULTILAYER MIE THEORY}

This section highlights the local field behavior of the excited nanoshell. This is relatively well known $[38,39]$, but we focus here on particularities particularly important in strong-field physics dealing with electron acceleration and collision: spatial amplitude, phase, and polarization inhomogeneities. With this in mind, we will extract optimum size and geometry parameters to be used in a strong-field experiment using femtosecond pulses centered at $800 \mathrm{~nm}$. We start with the monochromatic response and then show effects related to femtosecond ultrashort pulse response.

\section{A. Monochromatic response}

The local field enhancement (LFE), defined as $\mathbf{L}(\omega, \beta, \mathbf{r})=$ $\mathbf{E}^{\mathrm{loc}}(\omega, \beta, \mathbf{r}) /\left|E_{0}(\omega)\right|$, has been calculated as a function of wavelength and the core/particle volumes ratio $\beta$. This gives us the possibility to define a spectral amplitude and phase of the field enhancement in order to observe spectral field reshaping due to the resonance. The strongest enhancement occurs for the $x$ component of the local field. We therefore first focus on this contribution $\left(L_{x}\right)$. Figure 2 represents the maximum intensity enhancement $\left|L_{x}\right|^{2}$ as a function of wavelength and $\beta$ parameter for different nanoshell size: $R_{\text {shell }}=a_{1}=20 \mathrm{~nm}$ in (a), $R_{\text {shell }}=60 \mathrm{~nm}$ in (b), and $R_{\text {shell }}=100 \mathrm{~nm}$ in (c). The dashed white lines are highlighting the $800 \mathrm{~nm}$ wavelength behavior from which the optimal $\beta$ has been chosen. White lines are line-outs along those dashed lines. For a comparison as a function of particle size, (d) shows the spectral line-outs
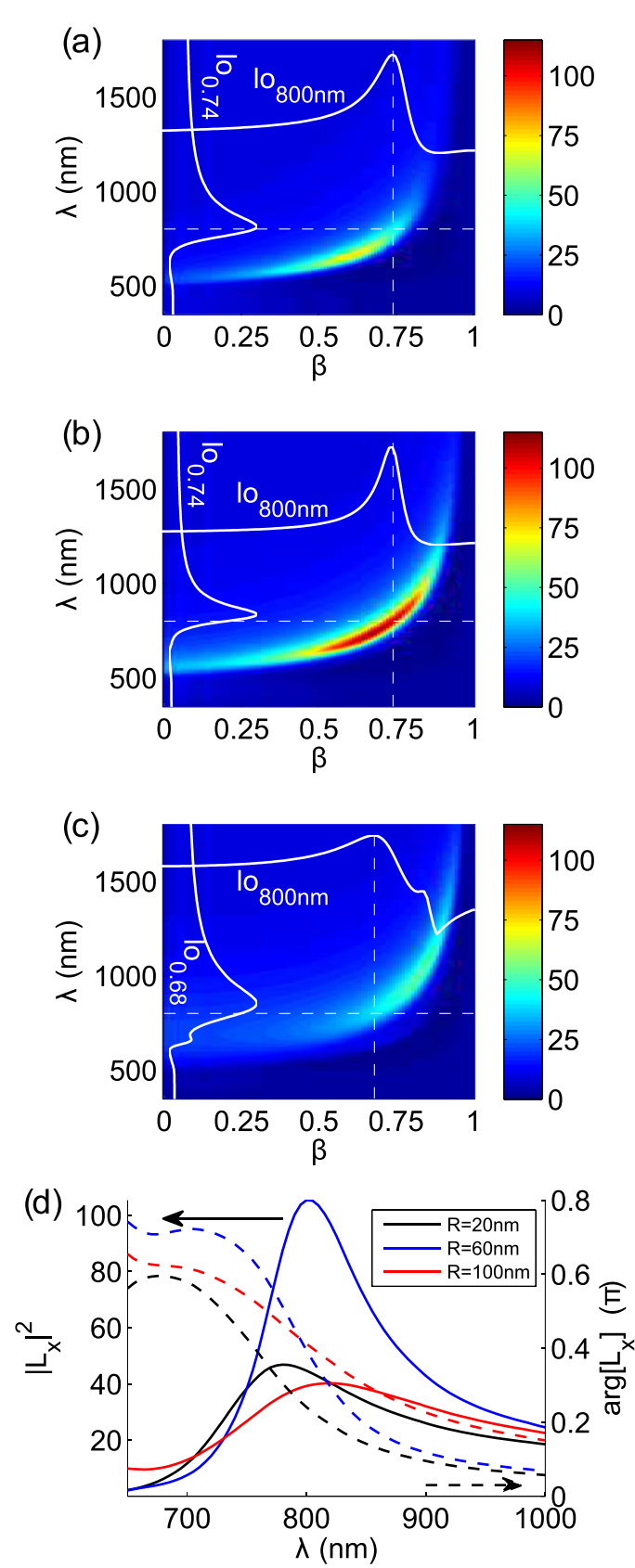

FIG. 2. (Color online) $\mathrm{SiO}_{2}$ core, gold shell intensity enhancement of the $x$ component (main contribution) as a function of wavelength and $\beta$ for (a) $R_{\text {shell }}=a_{1}=20 \mathrm{~nm}$, (b) $R_{\text {shell }}=60 \mathrm{~nm}$, and (c) $R_{\text {shell }}=100 \mathrm{~nm}$. The dashed white lines are highlighting the $800 \mathrm{~nm}$ wavelength behavior from which the optimal $\beta$ has been chosen. White lines are line-outs along those dashed lines. (d) Spectral intensity enhancement and phase in units of $\pi$ for the optimum $\beta$ of each nanoshell size.

of the intensity of the field enhancement (solid lines) and its phase (dashed lines) in units of $\pi$.

The effect of electron scattering on the shell surfaces (see Sec. II) is clearly visible on the broader width of the spectral intensity for small particles $\left(R_{\text {shell }}=20 \mathrm{~nm}\right)$ compared to bigger ones $\left(R_{\text {shell }}=60 \mathrm{~nm}\right)$. The LFE amplitude increases with the shell radius while the resonance bandwidth decreases. However at $100 \mathrm{~nm}$ there is a complete difference. The 
resonance bandwidth is even broader than the smallest particles and the LFE is decreasing again. This modification of behavior is related to the multipolar response of the particle. A radius of $100 \mathrm{~nm}$ is no longer small enough compared to the incident wavelength.

Choice of the optimum nanoshell size is made in terms of amplification of temporally short and spectrally broadband pulses. The optimum choice would be a broad and high LFE amplitude while keeping the phase nearly constant. This is not possible around resonances, and a tradeoff must be chosen. On the first hand, amplification at $800 \mathrm{~nm}$ is best for particles with a radius on the order of $60 \mathrm{~nm}$, but on the second hand, the resonance width is limited compared to bigger or smaller particles. This will induce an inherent amplitude reshaping of the local field with respect to the excitation. Moreover, when crossing the resonance an almost $\pi$ spectral phase jump is applied to the amplified field [see Fig. 2(d), dashed lines], elongating it temporally. On the contrary, smaller particles exhibit a smoother and much lower than $\pi$ phase jump, limiting this temporal spread. From this qualitative analysis, it seems that a balance has to be found on the particle size in terms of amplification and temporal reshaping. A good compromise in that respect would be to use particles of radius on the order of $60 \mathrm{~nm}$ whose resonance still possesses a $100 \mathrm{~nm}$ bandwidth and lead to the highest LFE at $800 \mathrm{~nm}$. We will show later that the phase variation can be corrected. We will therefore focus our study on these parameters: $\lambda=800 \mathrm{~nm}, R_{\text {shell }}=60 \mathrm{~nm}$, and $\beta=0.74$.

The LFE distributions $L(\omega, \beta, \mathbf{r})$ are shown in Fig. 3 for the chosen case $(\lambda=800 \mathrm{~nm}, \beta=0.74)$. The first observation is that the local field has a complex vectorial behavior with quite high values of $y$ and $z$ component even if the excitation field is linearly polarized along $\mathbf{u}_{\mathbf{x}}$. That behavior can have a significant impact on strong-field phenomena such as on the quivering motion of electrons after ionization. This will be discussed in Sec. IV on the classical electron trajectories.

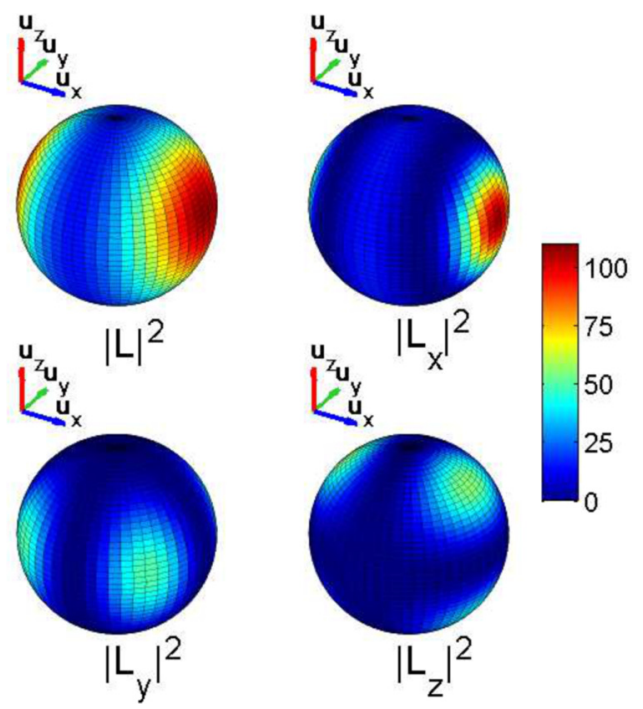

FIG. 3. (Color online) Total and partial local field enhancement on the nanoshell surface as a function of $\theta$ and $\phi$ for $\lambda=800 \mathrm{~nm}$ and $\beta=0.74$.
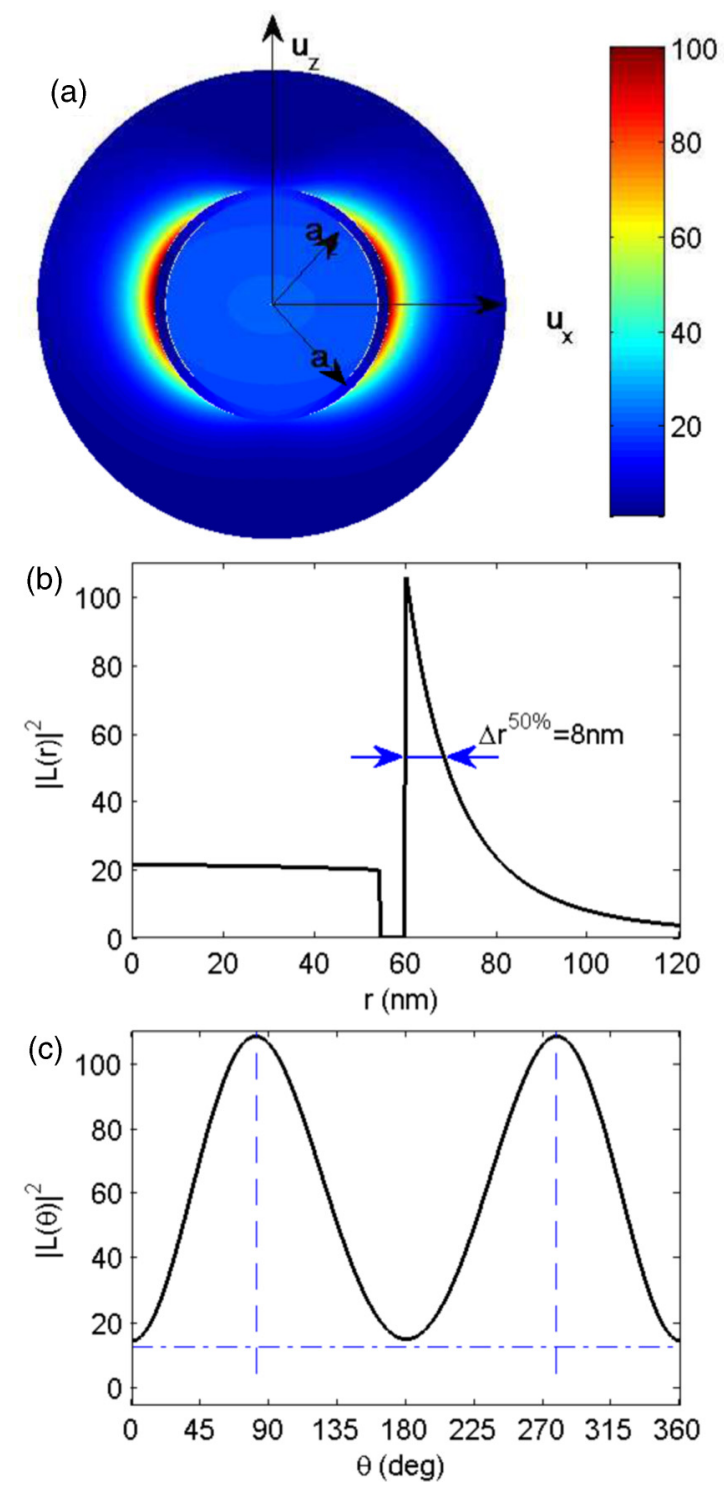

FIG. 4. (Color online) (a) Local intensity enhancement $|L(\mathbf{r})|^{2}$ in the $(\mathrm{O} x z)$ plane. (b) Line-out of $|L(\mathbf{r})|^{2}$ for $\theta=81.5^{\circ}$. (c) Line-out of $|L(\mathbf{r})|^{2}$ for $r=60 \mathrm{~nm}$.

Figures 4, 5, and 6 help to grasp the local behavior of the enhanced electric field by showing, respectively, its spatial amplitude, phase, and polarization properties. Figure 4(a) shows a cut of the $3 \mathrm{D}|L(\mathbf{r})|^{2}$ distribution in the $(\mathrm{O} x z)$ plane. The LFE is maximum around the laser polarization $\mathbf{u}_{\mathbf{x}}$ and is quickly decreasing from the shell edge and for increasing radius. Figure 4(b) shows a line-out of the distribution, along the direction $\theta=81.5^{\circ}$ where the enhancement is maximum. The intensity enhancement is nearly constant in the core, equal to about 20 , then it drops to zero in the metallic shell, and then reaches its highest value, $\approx 105$, on the outer face of the shell. It is quickly decreasing with increasing radius, with a $50 \%$ reduction for an increase of radius of $\Delta r^{50 \%} \approx 8 \mathrm{~nm}$. This quantity will be referred to as the decreasing field length. Figure 4(c) represents a similar line-out for $r=60 \mathrm{~nm}$ as a function of the polar angle $\theta$. The LFE is maximum at $\theta=81.5^{\circ}$ and $278.5^{\circ}$ (see vertical 

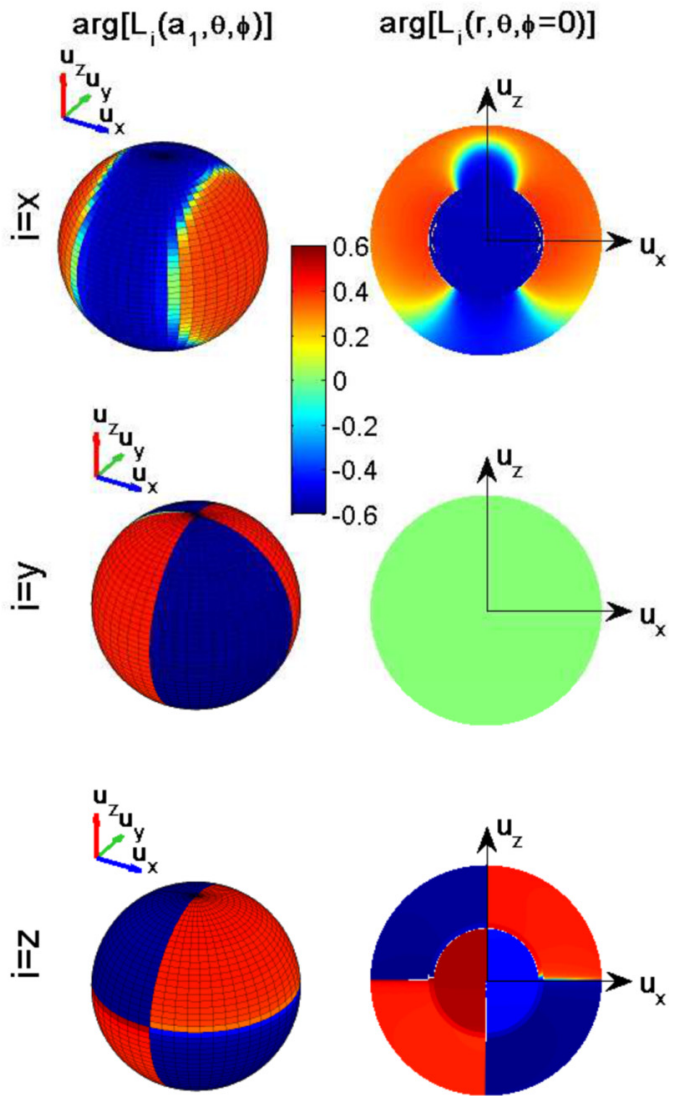

FIG. 5. (Color online) Local field enhancement spatial phase variation in units of $\pi$. Left column: distribution for $r=a_{1}$. Right column: spatial cut in the $(\mathrm{O} x z)$ plane.

dashed lines). This slight forward asymmetry is visible as well between $\theta=0^{\circ}$ and $180^{\circ}$ with higher LFE at $0^{\circ}$. This is due to the constructive/destructive interference between the incident wave and the local plasmonic field due to the evolution of the incident field spatial phase $(\phi=k z)$ that is not constant over the size of the nanoshell.

Figure 5 shows the spatial phase variation for each field component in units of $\pi$. The left column represents this phase at the surface of the nanoshell as a function of the spherical angles, $\theta$ and $\phi$, while the right column is a cut in the $(\mathrm{O} x z)$ plane $(\phi=0)$. It is clearly visible that almost no spatial phase variation is happening on the $x$ component in the region where the LFE is significant. The forward LFE asymmetry due to fields interferences is also clearly visible on the phase profile of the $x$ component. However, both the $y$ and $z$ component have $\pi$ phase shift with respect to the (Oyz), (Oxy), and (Oxz) planes. This phase behavior is reflected on the vectorial nature of the local field as represented in Fig. 6 highlighting the LFE in the (Oxz) plane in (a) and in the $x=R_{\text {shell }}$ plane in (b). The direction of the arrows shows the direction of the local field, and the length of the arrow shows its magnitude. The local field is parallel to the incident field around the $(\mathrm{O} x)$ axis, but as soon as one moves from it, the field acquires a strong transverse component along $(\mathrm{O} y)$ and a strong longitudinal component along $(\mathrm{O} z)$. This could have an important impact effect for electron tunnel ionization and rescattering [40] and (a)

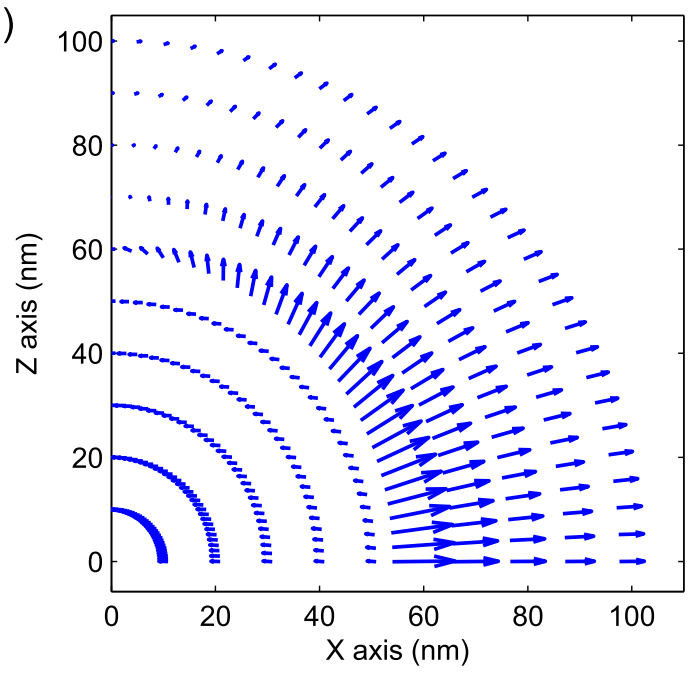

(b)

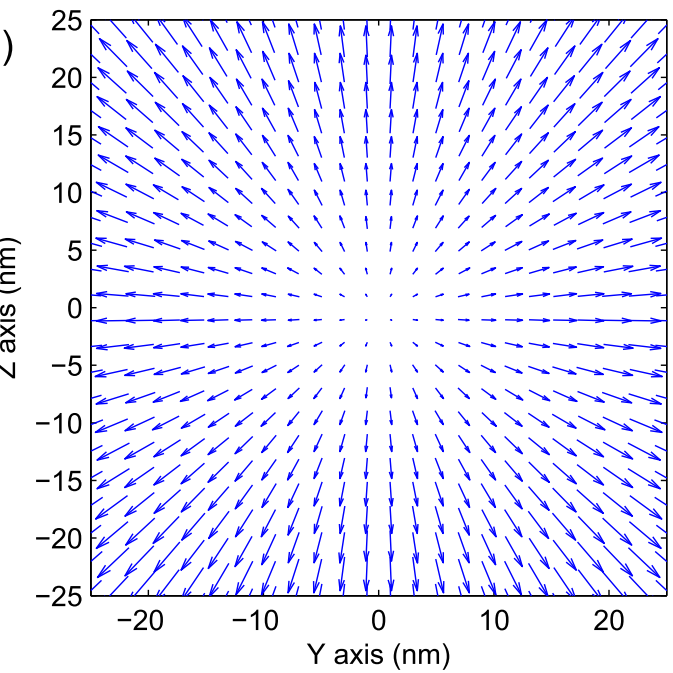

FIG. 6. (Color online) (a) Cut in the (Oxz) plane showing the direction and magnitude of the local electric field. (b) cut for $x=$ $R_{\text {shell }}$.

also modify the common "simple man's model" of harmonic generation [13].

The conclusion from this first study is that in terms of intensity, plasmonic field enhancement can be used to reach the strong-field regime using only low peak power lasers. Indeed an intensity amplification factor of about 100 has been calculated at the surface of the nanoshell. Using pulses with as low as $10^{11} \mathrm{Wcm}^{-2}$ would be enough to reach strong-field thresholds in gases and in metals. However the peculiar properties of the local field make a huge modification to standard laser only strong-field physics. The first modification is related to spatially inhomogeneous LFE. A recent study on gold tips pointed out the quenching of the electronic quiver motion after tunnel ionization [19] due to quickly spatially decreasing local enhanced field. If the electron excursion is on the order of the field decreasing length, then the field strength is not enough to modify the electron trajectory so that it will rescatter on the parent atom/nanoshell when the incident field is changing its direction. In that respect many 
theoretical studies looked at the spatially inhomogeneous field enhancement $[25,41,42]$ incidence on the above threshold ionization and high harmonic generation, however no study pointed out the complex vectorial nature of local fields. First the local field it is not transverse and does not have a homogeneous linear polarization in space. This will produce complicated electronic trajectories (see Sec. IV) and eventually high harmonic depolarization [43]. These inhomogeneities could, however, add some degree of control in the electron trajectory if the excitation field is shaped in polarization such that it modifies the vectorial properties of the local field.

\section{B. Ultrashort pulse response}

Another important aspect for strong-field physics and femtosecond lasers in general is the amplitude and phase spectral modulation due to the plasmonic resonance. It is well known, for instance, that propagating an ultrashort pulse in a glass plate will induce through dispersion a linear chirp increasing drastically both its overall temporal duration and its spectrotemporal properties. This section aims therefore to highlight the amplitude and phase reshaping of short pulses by plasmonic resonance in the nanoshell case and validate its use for ultrashort pulse enhancement.

Using selected parameters $R_{\text {shell }}=60 \mathrm{~nm}$ and $\beta=0.74$ from the last section, the nanoshell behavior excited by a temporally short pulse is simulated. Calculations are made using the previously mentioned generalized Mie theory and by adding coherently plane waves in the range $\lambda=600-1100 \mathrm{~nm}$ (see Sec. II). We verified that all local field components follow the same spectrotemporal properties. We are therefore highlighting this reshaping study to the stronger field component $E_{x}$ at $\theta=90^{\circ}$ and $r=R_{\text {shell }}$ to describe how the resonance phase impacts the temporal shape.

Figure 7 represents the spectrotemporal properties of the $x$ component of the local field. Spectra are shown in panel (a). The purple line is the spectral response of the nanoshell (thick lines are normalized amplitudes and dotted ones are the spectral phase in units of $\pi$ ). The black line is the Gaussian envelope of the excitation field (with a flat phase equal to zero). The spectrum is centered at $800 \mathrm{~nm}$ and has a FWHM width in intensity $\Delta \lambda=200 \mathrm{~nm}$ giving a temporal duration of about 5 fs. The blue line is the resulting field (product of the incoming field and the nanoshell response in the spectral domain) sharing the same phase as the nanoshell response. The locally enhanced field is reshaped both in amplitude and phase due to the plasmonic resonance. The width of the resonance cannot support such a large bandwidth and restricts it to about $92 \mathrm{~nm}$, while the spectral phase is showing a phase jump of almost $\pi$ across the spectrum.

The temporal field behavior is represented in panel (b) and corresponds to the spectra shown in panel (a). The incoming pulse is very short (black line $\Delta t=4.7 \mathrm{fs}$ FWHM in intensity), while the resulting local field has a long tail increasing its duration by almost a factor two (blue line $\Delta t=7.8 \mathrm{fs}$ ) and is retarded by slightly more than one period. Details about the spectrotemporal features are well represented by a spectrogram type Wigner plot [44] shown in panel (c). The spectral axis is vertical and the horizontal one represents time. Deviations from a temporally symmetric Fourier limited
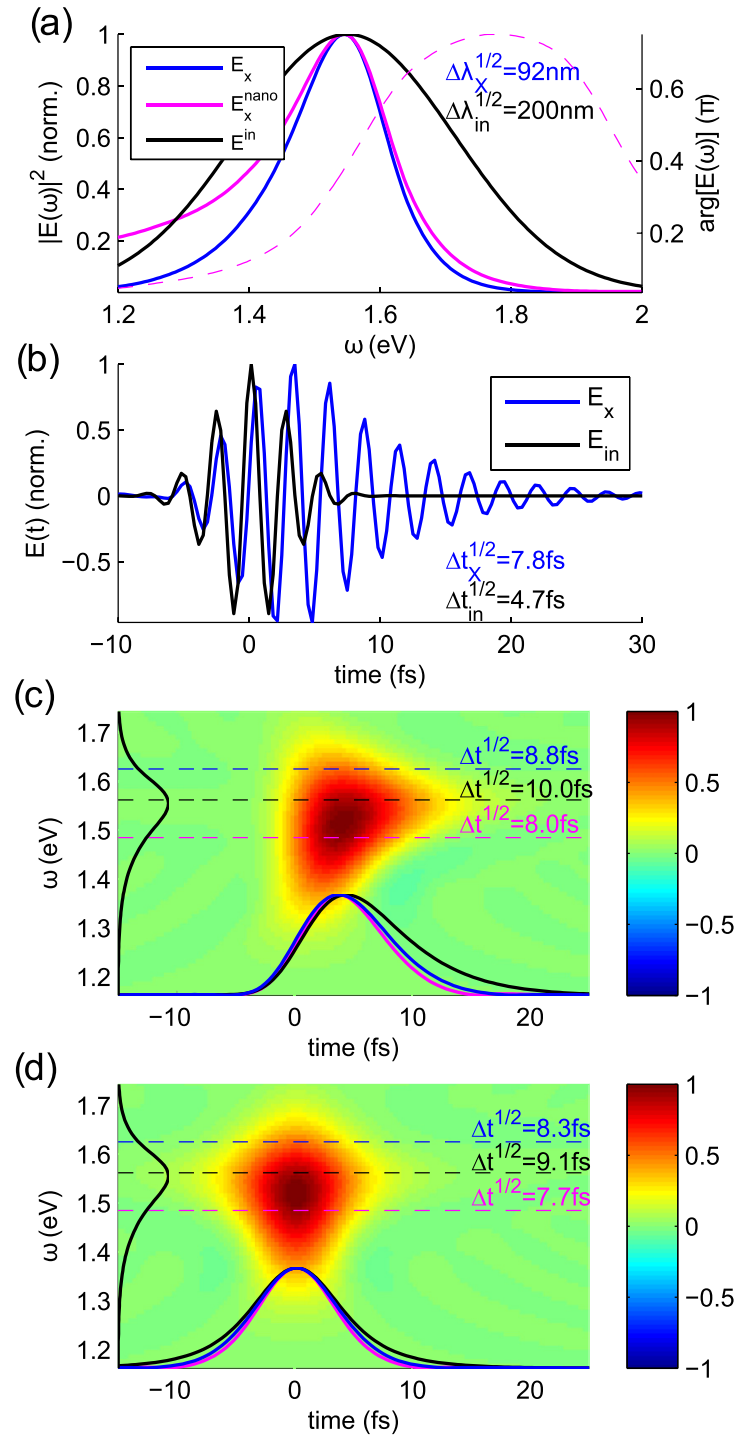

FIG. 7. (Color online) Spectrotemporal properties of the local field enhancement $L_{x}$. (a) Spectral amplitude (thick lines) and phase (dashed lines) of incident field (black) and spectral response of the nanoshell (purple). Their product gives the local field spectral amplitude (blue). The incident field phase is equal to zero. (b) Temporal profile of the local field corresponding to the spectra in panel (a). (c) Wigner representation of the local field (corresponding to blue curves in panels (a) and (b). (d) As in panel (c) but for an incident field spectral phase correcting for the nanoshell spectral phase (see text for details).

pulse are clearly visible. Moreover, all spectral components don't behave the same and the temporal duration is therefore spectrally dependent. Despite this, the effect is not very deleterious with less than 2 fs difference across the spectrum. Indeed, the phase variation in panel (a) is rather smooth and mostly linear over the reshaped spectrum leading to a delayed, almost Fourier limited pulse.

This slightly asymmetric temporal response can be further controlled applying a spectral phase shaping on the incident pulse. Panel (d) shows a Wigner plot of the local field excited by a laser pulse having the same spectral amplitude as in panel (a) but a spectral phase opposite to the one created by the 
plasmonic resonance. The temporal asymmetry is washed out and the pulse is the shortest possible given the bandwidth. This shaping is optimum only at the position where the local field has been calculated. If the spectral response is spatially inhomogeneous then the local field cannot be controlled everywhere. Fortunately, in the nanoshell case this phase shaping is adapted in all the areas where the local field enhancement is maximum.

This a priori phase shaping could be applied to systems where reshaping is much stronger or simply used to coherently control local field spectrotemporal behavior. Even more complex shaping could be envisaged such as polarization shaping [45] or multicolor excitations [46].

\section{CLASSICAL ELECTRON TRAJECTORY}

In strong-field physics and especially in the framework of the strong-field approximation, electrons tunnel ionized are following specific quantum orbits. These can in some extent be approximated by classical calculation of an electron accelerated by the local field. This approximation gives an idea of whether the electron may or not recollide. In plasmonic field enhancement on nanotips, it has been shown [19] that above a certain excursion length, the spatially decaying field is not intense enough to modify the electron trajectory that cannot be driven back toward the emitter. That effect and the complex polarization nature of the local field are tested in this section. We suppose that electrons are born in the close vicinity of the nanoshell surface. This could model both a tunnel ionization from the particle itself or from an atom lying close to the nanoshell. The electron is only subject to the local field, and we neglect the particle or ion influence on its trajectory. The tunnel ionization rate is not taken into account; only the trajectory behavior is highlighted here. Trajectories have been calculated for two driving wavelength: 800 and $1300 \mathrm{~nm}$. The excursion will be higher in the $1300 \mathrm{~nm}$ case, while the field decreasing length is mostly governed by the particle size and is therefore constant. The modification of trajectory behavior between those two cases will highlight the spatial inhomogeneities related to plasmonic enhancement.

Figure 8 shows electron trajectories calculated using classical Newton equations for different initial positions around the nanoshell $(0.1 \mathrm{~nm}$ from the surface) and for a driving wavelength of $800 \mathrm{~nm}$ in panel (a) and $1300 \mathrm{~nm}$ in panel (b). In each panel two particular trajectories have been zoomed in to visualize their details, especially the shift along the $(\mathrm{O} z)$ axis. Temporal evolution of the field has been calculated as described in the previous section. As shown in Fig. 2(b), the enhancement at $1300 \mathrm{~nm}$ is much lower than at $800 \mathrm{~nm}$ (respectively, $\left|L_{x}^{\max }\right|^{2}=30$ and $\left|L_{x}^{\max }\right|^{2}=105$ ), however in both cases we supposed a local maximum intensity of $I_{\max }=$ $1 \times 10^{14} \mathrm{Wcm}^{-2}$ in order to visualize only the wavelength effect on trajectories. Practically, the $1300 \mathrm{~nm}$ incident field intensity would be increased relative to the $800 \mathrm{~nm}$ one so that local field maxima are equal in both cases.

For simplicity, and with the same conclusion, only 2D trajectories in the $(\mathrm{O} x z)$ plane have been calculated (in this plane, the $y$ component of the field is nearly zero so as to justify this approximation, see Fig. 3). We supposed that the electron tunneled out of the nanoshell at different positions
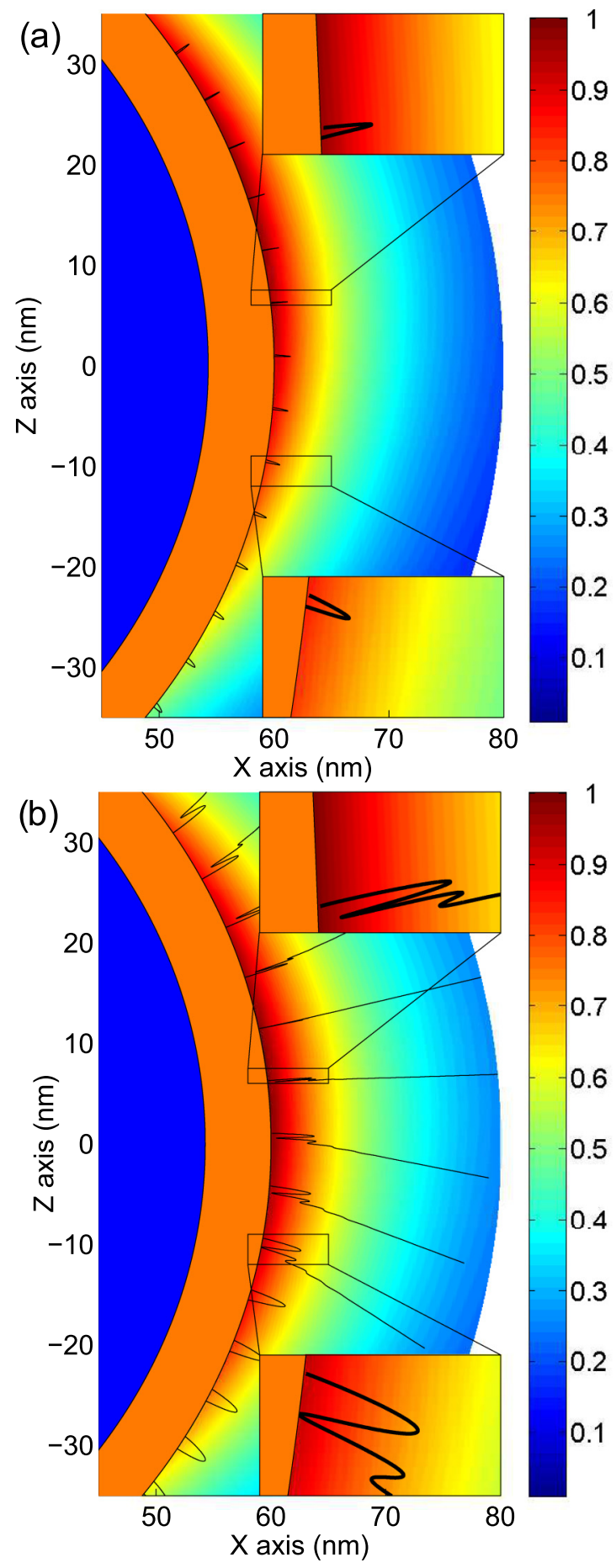

FIG. 8. (Color online) Set of electronic trajectories around a nanoshell excited with $I_{\max }^{\text {loc }}=1 \times 10^{14} \mathrm{Wcm}^{-2}$ at $800 \mathrm{~nm}$ in (a) and $1300 \mathrm{~nm}$ in (b). Rectangles are zooms of selected trajectories. The color scale represents the field intensity spatial evolution in units of $I_{\max }^{\mathrm{loc}}$. Blue and orange areas are, respectively, core and shell.

without any initial velocity and with a time of birth slightly after the field maximum. Results are clear: For the $800 \mathrm{~nm}$ driving wavelength, the electron excursion is small enough so that recollision on the nanoshell can occur at all positions. At $1300 \mathrm{~nm}$ this is no longer the case. Electrons travel approximately $3 \mathrm{~nm}$ before the field switches its direction, and 

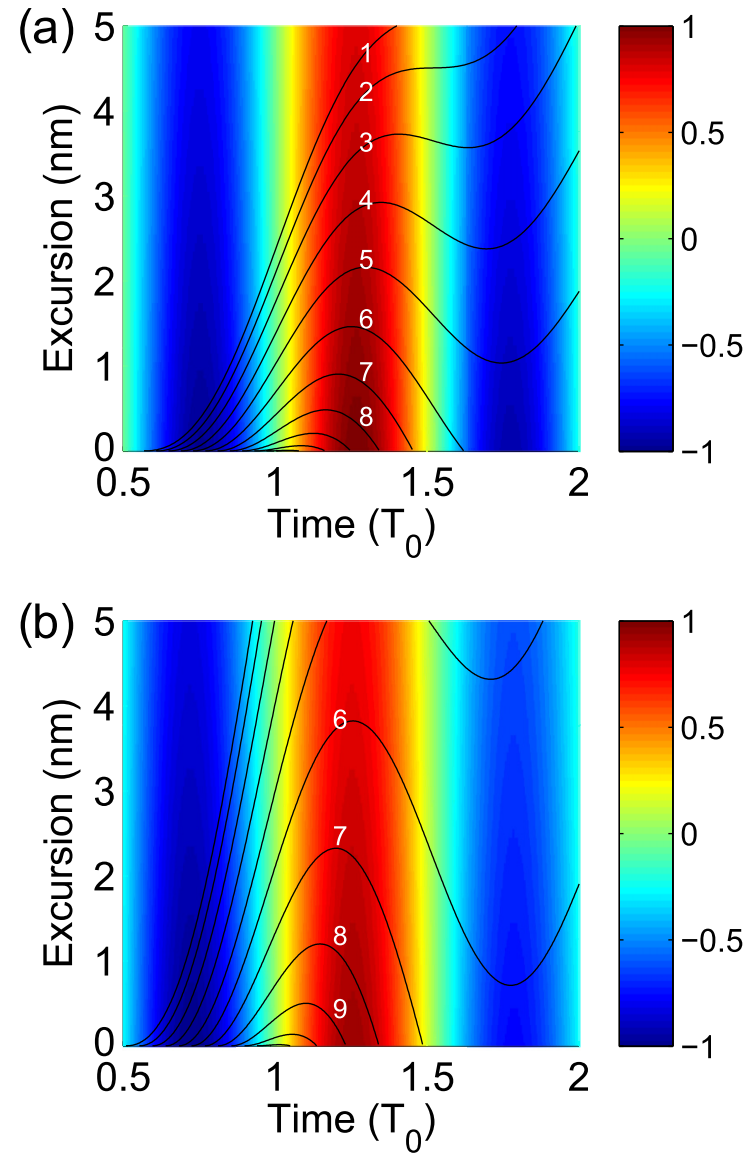

FIG. 9. (Color online) Electron excursion as a function of time of birth within the local field (in units of field period) for (a) $\lambda_{0}=800 \mathrm{~nm}$ and (b) $\lambda_{0}=1300 \mathrm{~nm}$. Electrons are born at $r=60.1 \mathrm{~nm}$ and $\theta=74^{\circ}$ for $800 \mathrm{~nm}$ and $\theta=82^{\circ}$ for $1300 \mathrm{~nm}$. The color scale highlights the driving electric field in units of maximum intensity $I_{\max }^{\text {loc }}=1 \times 10^{14}$ $\mathrm{Wcm}^{-2}$.

at that position it is too weak to pull back the electron. Because of these longer trajectories, electrons are more sensitive to spatial field direction changes. While at $800 \mathrm{~nm}$, trajectories are mostly linear, at $1300 \mathrm{~nm}$ the shift in the $(\mathrm{Oz})$ direction is much more important up to about $1 \mathrm{~nm}$ (see Fig. 8 bottom zooms). It is important to note that recollision will happen on a different site from the electron birth's one. This shows that recollision on hypothetical atoms placed close to the shell is unlikely to occur if the side displacement is higher than the atom size.

The field maximum position on the nanoshell surface is dependent of the driving wavelength. For a $800 \mathrm{~nm}$ center wavelength, this maximum is located on the surface at $\theta=74^{\circ}$ and at $\theta=82^{\circ}$ for $1300 \mathrm{~nm}$. For those angles, trajectories are almost linear and can be characterized only by the excursion radius with respect to the initial position. Figure 9 shows a sample of trajectories calculated for incident fields centered at $800 \mathrm{~nm}$ in panel (a) and at $1300 \mathrm{~nm}$ in panel (b). Time and space evolution of the local field for each particular angle is represented by the color scale in units of maximum field intensity $I_{\max }^{\text {loc }}=1 \times 10^{14} \mathrm{Wcm}^{-2}$. Birth time of electrons are set as fractions of the field period and distributed around a field maximum for both wavelength. The corresponding electron trajectories are labeled by white numbers on the figure. The influence of the wavelength is striking; excursions are much broader for the $1300 \mathrm{~nm}$ case. However because of the relatively low field decreasing length compared to electrons excursions, the recolliding trajectories are almost the same in both cases (only trajectory six is not recolliding in the $1300 \mathrm{~nm}$ case). This effect would be much stronger if the driving wavelength was much higher so that the electron excursion would be of the order of the decreasing length, that is about $8 \mathrm{~nm}$. A much more detailed and complex calculation would be needed to give quantitative results, but these classical trajectories shown in Figs. 8 and 9 highlight perfectly the complex local field nature around nanostructures.

\section{CONCLUSION}

This study shows that plasmonic enhancement can be used to reach the strong-field domain using a simple femtosecond oscillator. Nanostructures' plasmonic resonance can be efficiently tailored to match the field wavelength. Nanoshells are particularly suited for strong-field experiment. Their isotropic shapes make them not sensitive to orientation with respect to the laser polarization. This means they could be used as a nanoparticle jet in vacuum just as atoms or molecules using aerodynamic lenses $[27,47]$. The volume ratio of the core with respect to the shell is an experimental parameter used to adjust the position of the plasmonic resonance leading to about two orders of magnitude enhancement in intensity at $800 \mathrm{~nm}$. However, local fields surrounding the nanoshell have a complex behavior in space, time, and polarization. The impact of such features for strong-field physics has been highlighted with classical electron trajectories, and the conclusion is that restricting the study to an $800 \mathrm{~nm}$ driving wavelength will make the chosen nanoshell parameters suitable for strong-field experiments. This model can serve as a basis for experimental data analysis but also for more complete theoretical development of strong-field physics taking into account highly inhomogeneous fields both in amplitude and polarization.

\section{ACKNOWLEDGMENTS}

The authors thank T. Auguste for fruitful discussions. This work has been supported by the "Triangle de la physique" Grant No. ATTOCONTROL 2012-046T.
[1] J. M. Pitarke, V. M. Silkin, E. V. Chulkov, and P. M. Echenique, Rep. Prog. Phys. 70, 1 (2007).

[2] J. A. Schuller, E. S. Barnard, W. Cai, Y. C. Jun, J. S. White, and M. L. Brongersma, Nat. Mater. 9, 193 (2010).
[3] D. K. Gramotnev and S. I. Bozhevolnyi, Nat. Photon. 4, 83 (2010).

[4] N. J. Halas, S. Lal, W.-S. Chang, S. Link, and P. Nordlander, Chem. Rev. 111, 3913 (2011).

[5] M. A. Garcia, J. Phys. D 45, 389501 (2012). 
[6] W. L. Barnes, A. Dereux, and T. W. Ebbesen, Nature (London) 424, 824 (2003).

[7] A. Cuche, O. Mollet, A. Drezet, and S. Huant, Nano Lett. 10, 4566 (2010).

[8] Z. Wang, P. Tao, Y. Liu, H. Xu, Q. Ye, H. Hu, C. Song, Z. Chen, W. Shang, and T. Deng, Sci. Rep. 4, 6246 (2014).

[9] A. Kinkhabwala, Z. Yu, S. Fan, Y. Avlasevich, K. Müllen, and W. E. Moerner, Nat. Photon. 3, 654 (2009).

[10] S. Nie and S. R. Emory, Science 275, 1102 (1997).

[11] M. P. Kreuzer, M. U. González, R. Quidant, I.-i. D. C. Fotoniques, and M. T. Park, ACS Nano 3, 1231 (2009).

[12] M. Ferray, A. L'Huillier, X. F. Li, L. A. Lomprk, G. Mainfray, and C. Manus, J. Phys. B 21, L31 (1988).

[13] P. Corkum, Phys. Rev. Lett. 71, 1994 (1993).

[14] K. C. Kulander, K. J. Schafer, and J. L. Krause, Super-Intense Laser-Atom Phys. NATO ASI Ser. 316, 95 (1993).

[15] M. Lewenstein, P. Balcou, M. Y. Ivanov, A. L'Huillier, and P. B. Corkum, Phys. Rev. A 49, 2117 (1994).

[16] Strong Field Laser Physics, Springer Series in Optical Sciences, Vol. 134, edited by T. Brabec (Springer, New York, 2009).

[17] P. Hommelhoff, C. Kealhofer, and M. Kasevich, Phys. Rev. Lett. 97, 247402 (2006).

[18] M. Kruger, M. Schenk, and P. Hommelhoff, Nature (London) 475, 78 (2011).

[19] G. Herink, D. R. Solli, M. Gulde, and C. Ropers, Nature (London) 483, 190 (2012).

[20] S. Kim, J. Jin, Y. Kim, I. Park, Y. Kim, and S. Kim, Nature (London) 453, 757 (2008).

[21] M. Sivis, M. Duwe, B. Abel, and C. Ropers, Nature (London) 485, E1 (2012).

[22] M. Sivis, M. Duwe, B. Abel, and C. Ropers, Nat. Phys. 9, 304 (2013).

[23] I.-Y. Park, S. Kim, J. Choi, D.-H. Lee, Y.-J. Kim, M. F. Kling, M. I. Stockman, and S.-W. Kim, Nat. Photon. 5, 677 (2011).

[24] M. F. Ciappina, S. S. Aćimović, T. Shaaran, J. Biegert, R. Quidant, and M. Lewenstein, Opt. Express 20, 26261 (2012).

[25] T. Shaaran, M. F. Ciappina, R. Guichard, J. A. Pérez-Hernández, L. Roso, M. Arnold, T. Siegel, A. Zaïr, and M. Lewenstein, Phys. Rev. A 87, 041402 (2013).

[26] J. Feist, M. Reid, and M. Kling, Phys. Rev. A 87, 033816 (2013).

[27] S. Zherebtsov, T. Fennel, J. Plenge, E. Antonsson, I. Znakovskaya, A. Wirth, O. Herrwerth, F. Süssmann, C. Peltz, I.
Ahmad, S. a. Trushin, V. Pervak, S. Karsch, M. J. J. Vrakking, B. Langer, C. Graf, M. I. Stockman, F. Krausz, E. Rühl, and M. F. Kling, Nat. Phys. 7, 656 (2011).

[28] J. Sinzig and M. Quinten, Appl. Phys. A Solids Surfaces 58, 157 (1994).

[29] S. Derom, A. Berthelot, A. Pillonnet, O. Benamara, A. M. Jurdyc, C. Girard, and G. Colas des Francs, Nanotechnology 24, 495704 (2013).

[30] A. Arbouet, F. Houdellier, R. Marty, and C. Girard, J. Appl. Phys. 112, 053103 (2012).

[31] C. F. Bohren and D. R. Huffman, in Absorption and Scattering of Light by Small Particles (Wiley-VCH Verlag GmbH, Weinheim, Germany, 1998), Chap. 4, pp. 82-129.

[32] P. B. Johnson and R. W. Christy, Phys. Rev. B 6, 4370 (1972).

[33] T. Stanislauskas, R. Budriunas, R. Antipenkov, A. Zaukevicius, J. Adamonis, A. Michailovas, L. Giniunas, R. Danielius, A. Piskarskas, and A. Varanavicius, Opt. Express 22, 1865 (2014).

[34] A. Moroz, J. Phys. Chem. C 112, 10641 (2008).

[35] M. G. Blaber, M. D. Arnold, and M. J. Ford, J. Phys. Chem. C 113, 3041 (2009).

[36] A. Monmayrant, S. J. Weber, and B. Chatel, J. Phys. B At. Mol. Opt. Phys. 43, 103001 (2010).

[37] A. Monmayrant and B. Chatel, Rev. Sci. Instrum. 75, 2668 (2004).

[38] P. Mélinon, S. Begin-Colin, J. L. Duvail, F. Gauffre, N. H. Boime, G. Ledoux, J. Plain, P. Reiss, F. Silly, and B. WarotFonrose, Phys. Rep. 543, 163 (2014).

[39] R. G. Chaudhuri and S. Paria, Chem. Rev. 112, 2373 (2012).

[40] H. Reiss, Phys. Rev. Lett. 101, 043002 (2008).

[41] M. F. Ciappina, T. Shaaran, and M. Lewenstein, Annalen der Physik 525, 97 (2013).

[42] T. Shaaran, M. F. Ciappina, and M. Lewenstein, Phys. Rev. A 87, 053415 (2013)

[43] P. Antoine, B. Carre, A. L. Huillier, and M. Lewenstein, Phys. Rev. A 55, 1314 (1997).

[44] J. Paye, IEEE J. Quantum Electron. 28, 2262 (1992).

[45] T. Brixner and G. Gerber, Opt. Lett. 26, 557 (2001).

[46] S. G. Rodrigo, H. Harutyunyan, and L. Novotny, Phys. Rev. Lett. 110, 177405 (2013).

[47] J. Shu, K. R. Wilson, M. Ahmed, and S. R. Leone, Rev. Sci. Instrum. 77, 043106 (2006). 